\title{
Rigidity and actuality-dependence
}

\author{
Jussi Haukioja
}

Published online: 3 November 2010

(C) The Author(s) 2010. This article is published with open access at Springerlink.com

\begin{abstract}
It is generally assumed that rigidity plays a key role in explaining the necessary a posteriori status of identity statements, both between proper names and between natural kind terms. However, while the notion of rigid designation is well defined for singular terms, there is no generally accepted definition of what it is for a general term to be rigid. In this paper I argue that the most common view, according to which rigid general terms are the ones which designate the same kind in all possible worlds, fails to deliver a posteriori necessities. I also present an alternative view, on which the work of explaining a posteriori necessities is not done by rigidity, but by a related metasemantic notion, which I call actuality-dependence.
\end{abstract}

Keywords Rigidity $\cdot$ Modality $\cdot$ Identity $\cdot$ Kripke

In Naming and Necessity (Kripke 1980), Saul Kripke convinced most of his readers that proper names are rigid designators, that is, that they designate the same individual in all possible worlds. From this observation the necessity of true a posteriori identities such as "Hesperus is Phosphorus" is thought to follow straightforwardly. Kripke (1980), and Putnam (1975), also claimed that some kind terms, most notably natural kind terms, are rigid as well. This is supposed to have theoretical consequences parallel to the ones concerning proper names: just as the rigidity of proper names plays a central role in showing that "Hesperus is Phosphorus" is necessary (if true), the rigidity of natural kind terms is supposed to

\footnotetext{
J. Haukioja $(\bowtie)$

Department of Philosophy, Norwegian University of Science and Technology,

7491 Trondheim, Norway

e-mail: jussi.haukioja@ntnu.no

J. Haukioja

Department of Behavioural Sciences and Philosophy, University of Turku, 20014 Turku, Finland
} 
entail that identity statements such as "Water is $\mathrm{H}_{2} \mathrm{O}$ ", "Heat is mean molecular kinetic energy", and "Tigers are animals"1 are also necessary (if true). In this paper I want to question this assumption and claim that, at least as a far as statements about natural kinds are concerned, the work of explaining the necessity of a posteriori identifications is really done by a notion I will call actualitydependence.

\section{Background}

The notion of rigidity for singular terms is straightforward: "Let's call something a rigid designator if in every possible world it designates the same object" (Kripke 1980, p. 48). If proper names are rigid, it follows (among other things) that identity statements between proper names are necessary if true. For example, given that "Hesperus" and "Phosphorus" are rigid designators, and given that they are coreferential in the actual world, it follows that they designate the same object in all possible worlds. As a consequence, "Hesperus is Phosphorus" is necessary if true, even though its truth or falsity can only be known a posteriori.

The rigidity of proper names does, then, seem to play a key role in the explanation of the necessity of a posteriori identity statements between names. We find out empirically that, in the actual world, Hesperus is Phosphorus; we observe that the two proper names are rigid; we can then deduce that the identity is necessary, though a posteriori. According to the received view, we can establish the necessity of a posteriori identifications according to the following schema, (NAP): ${ }^{2}$

(NAP1) Discover empirically that, in the actual world, $\boldsymbol{A}=\boldsymbol{B}$ is true. ${ }^{3}$

(NAP2) Note that A and B are rigid.

(NAP3) Reason from (NAP1) and (NAP2) that $\boldsymbol{A}=\boldsymbol{B}$ is necessary.

It is generally assumed (at least implicitly) that (NAP) works regardless of whether $\mathrm{A}$ and $\mathrm{B}$ are proper names or natural kind terms.

The problem with applying (NAP) to general terms is that it is not at all clear what it $i s$ for a general term to be rigid. Kripke only gave a definition of rigidity for singular terms, and despite the rather extensive (and still growing) literature on the question, no generally accepted definition of rigidity for general terms has been found.

\footnotetext{
1 The last example is, of course, not an identity. For simplicity, I will follow Soames (2002, p. 255) in counting sentences of this sort as theoretical identity statements as well, since they are thought to have a similar modal and epistemic status, and the rigidity of the natural kind terms that appear in them is assumed to play the same kind of role in explaining their necessity.

2 (NAP) is not intended to be a deductively valid argument, and we may well need additional essentialist premises to make it into one (cf. Salmon 1982). Be that as it may, (NAP1) and (NAP2) will still need to be included, which is enough for my purposes in this paper. I should also add that there are theorists who explicitly reject (NAP), for example Martí (2004). Nevertheless, (NAP) is clearly a very widely assumed schema.

${ }^{3}$ I am using boldface italics as corner quotes.
} 
It is often assumed that general terms designate their extensions, but combining this assumption with Kripke's definition will clearly not do. We would then be claiming that rigid general terms have the same extension across possible worlds, and it is easy to see the problem. On this view, most general terms, including natural kind terms, would come out non-rigid. For example, there could be more or fewer cats than there actually are.

A more promising idea is the claim that rigid general terms ascribe to objects properties which are possessed essentially (Cook 1980; Devitt 2005; GómezTorrente 2006). ${ }^{4}$ Some of Kripke's comments on proper names do seem to lead into this direction, but as a criterion of rigidity for general terms, the essentialist view runs into two kinds of problems. Firstly, it has been argued (Soames 2002, pp. 256-259) that the view cannot deliver necessities of the right sort. I am not going to discuss this problem here (see Gómez-Torrente 2006 for a reply); in my opinion, the second problem is severe enough to make the essentialist view highly unattractive. This problem is based simply on the observation that, if the essentialist view were correct, many terms which have been considered at least serious candidates for rigidity would be obviously non-rigid: take, for example, terms such as "red" and "hot", both of which are listed as rigid by Kripke (1980, p. 134). There may be room for discussion about whether colour terms in fact are rigid, but if the essentialist view were correct, there should be no doubt whatsoever about their status: in general, red things are obviously not essentially red. Hence, the essentialist notion of rigidity simply cannot be the notion that has been presupposed in these discussions. We should at least see if we can do better.

\section{The 'Simple proposal'}

I will now turn to the view that is by far most commonly held (or implicitly assumed) on this question. The view I have in mind is the "simple proposal" (henceforth: SP), versions of which are held by a number of authors (LaPorte 2000, 2004, 2006; Martí 2004; Salmon 2005; Linsky 2006; López de Sa 2008; I'm taking the label for the view from López de Sa). While the views of the proponents of SP diverge on some important issues, they agree on roughly the following definition of rigidity for general terms:

(SP) $\mathrm{x}$ is a rigid general term iff $\mathrm{x}$ designates the same kind in all possible worlds

Something like this view seems to be often assumed in discussions of natural kinds and the necessary a posteriori, and it would be hard to deny the attractiveness of this proposal. Moreover, it is easy to come up with examples which seem to support SP (such as "red" vs. "Mary's favourite colour", to take a well-worn example).

However intuitive SP may seem, it has been subject to heavy criticism. I will now take a brief look at two existing objections that have been raised against SP.

\footnotetext{
${ }^{4}$ More precisely: "a general term ' $F$ ' is a rigid applier iff it is such that if it applies to an object in any possible world, then it applies to that object in every possible world in which the object exists." (Devitt 2005, p. 146).
} 
I do not think they succeed in refuting SP, but a quick review of these problems will be useful in bringing out commitments and consequences of the view; these will play a role in my own argument against SP, which I will present in Sect. 2.2.

\subsection{Old Objections to SP: trivialization and over-generalization}

The first objection is that SP trivializes the notion of rigidity, by making all kind terms rigid (Schwartz 2002; Soames 2002). After all, unless we place restrictions on which kinds are real kinds (and thus legitimate), there will be a kind corresponding to any kind term. Consider the example mentioned above, "Mary's favourite colour". Proponents of SP would naturally want to say that it is non-rigid, because it might designate one colour in this world and another colour in another world. But why say this? Why not say, instead, that it designates the same kind in all worlds: the kind Mary's-favourite-colour?

Defenders of SP have sought a reply along the following lines (I am here mostly following Martí 2004). First, we are to suppose that general terms designate, when evaluated at a world, a function from possible worlds to objects. Rigid general terms will then be the ones which designate the same function at all worlds. On this elaboration of SP, "Mary's favourite colour" would be non-rigid, because, at $w_{1}$, it might designate the function from worlds to the red objects, whereas at $w_{2}$, it might designate the function from worlds to the blue objects, and so on.

In fact, it turns out that this commitment alone will not save SP from the trivialization objection (see López de Sa 2007)—SP also needs to provide linguistic data of a certain sort to avoid a further problem. But in fact data of the sort needed does seem to exist (LaPorte 2006; Martí and Martínez 2007). I will not discuss this issue here, but merely assume that this line of response will work. It is worth noting, however, that to deal with the trivialization objection SP needs to assume that general terms designate abstract objects such as functions (rather than their extensions, as some would like to hold on independent grounds). This assumption will play a role in my own argument against SP.

The second objection is that, even if SP does not trivialize the notion of rigidity by making all kind designators rigid, it will still over-generalize by making far too many of them rigid. For example, it seems to follow straightforwardly from SP that terms such as 'bachelor' and 'hunter' are rigid. 'Bachelor' rigidly designates the kind bachelor-the function from worlds to all and only the bachelors-and similarly for 'hunter', and any other simple general term. For some (e.g. Soames 2002; Schwartz 2002), this is reason enough to reject SP, but the proponents of SP are quite happy to accept the consequence. On SP, rigidity is not a feature that is exclusive to natural kind terms (and perhaps a few others). Most general terms are rigid (indeed, it appears that only unrigidified definite descriptions of kinds will turn out non-rigid.) It does not seem very fruitful to carry on a debate about whether this is an acceptable conclusion. For my purposes here, it is enough that we make note of this conclusion, because it will play a role in my argument against SP, to which we shall now turn. 


\subsection{A new objection to SP: theoretical role}

My own objection to SP could be called the objection from theoretical role. The notion of rigidity for general terms was supposed to play a central role in explaining the necessity of a posteriori identifications between rigid general terms. And most ${ }^{5}$ proponents of SP claim that their view does just that. LaPorte, for example, gives a nice example of an a posteriori identity between kind terms: "Brontosaurus = Apatosaurus". This example has a history quite similar to "Hesperus is Phosphorus": Brontosaurus and Apatosaurus were first thought to be two distinct species of dinosaur, but this was later empirically disconfirmed (see LaPorte 2004, p. 37). ${ }^{6}$ LaPorte claims that rigidity, as elaborated in SP, plays a role in explaining the fact that this identity is necessary, even though it is a posteriori. "Brontosaurus" and "Apatosaurus" were discovered to denote the same kind, and because they are rigid, they denote the same kind in all worlds, and the identification is shown to be necessary (LaPorte 2004, p. 40).

While this explanation-which is clearly an instance of the schema (NAP) - may seem to work, and even be obvious, I will argue that it in fact fails. ${ }^{7}$ But first, we should note that in the case of kind terms, the identity discovered in (NAP1) can be understood in two very different ways. This is perhaps easiest to see with the most common example of such an identity statement, "Water is $\mathrm{H}_{2} \mathrm{O}$ ". This statement can be read in two ways: as a quantified biconditional, or as a true identity statement between kinds. I will now look at each reading and claim that SP fails to play the required role in delivering the necessary a posteriori status of these identities, regardless of which reading we adopt.

First, suppose that the purported identity is read as a quantified biconditional (or as a quantified conditional; this is the natural reading of another common example, "tigers are animals"):

$$
\forall x\left(x \text { is water } \leftrightarrow x \text { is } \mathrm{H}_{2} \mathrm{O}\right)
$$

On this reading, the kind terms function as predicates, and the discovery in (NAP1) will be a discovery about actual co-extension: we discover that, in the actual world, anything composed of water is composed of $\mathrm{H}_{2} \mathrm{O}$, and vice versa.

It is fairly easy to see that, on this reading of theoretical identities, SP runs into problems. Recall that, as we saw in connection with the overgeneralization problem, according to SP most kind terms are rigid. For example, "renate" and "cordate"

\footnotetext{
5 A notable exception is Martí (2004), who claims that it was a mistake to expect rigidity to play such a role in the first place. In my view, this is really to give up on what most people have taken to be theoretically interesting about rigidity. So, at the very least, it is worth asking whether there is a semantic feature which does play such a role, whether or not it is justifiable to label it rigidity.

6 It is unfortunate that the standard example of an a posteriori necessity between kinds, "Water is $\mathrm{H}_{2} \mathrm{O}$ ", is not fully analogous to "Hesperus is Phosphorus" in this respect. In Haukioja (2006) I use a similar example involving species of butterfly, "Araschnia levana = Araschnia prorsa".

7 Somewhat different kinds of doubts about SP's ability to deliver a posteriori necessities have been expressed by Stephen Schwartz (2002, pp. 270-272); for a reply, see LaPorte (2006).
} 
will quite obviously come out rigid on SP, rigidly denoting the properties of having a kidney and having a heart, respectively. ${ }^{8}$ But now, if in the actual world all and only renates are cordates, (NAP) should apply to the biconditional "renates are cordates" as well: (NAP1) and (NAP2) are satisfied, so it should follow that it is a necessary a posteriori truth. But that cannot be right: even if all and only renates should actually turn out to be cordates, it appears obvious that there could be creatures that only have a kidney or a heart, but not both. "Renates are cordates" is contingently true (if true at all). The upshot is that SP's notion of rigidity cannot play the role of delivering, via schema (NAP), the necessity of true theoretical identifications of the quantified biconditional type, since it would confer necessity to biconditionals which quite clearly are contingent.

Suppose, on the other hand, that the identity in (SAP1) is a true identity between kinds (This reading seems natural with some standard examples, such as "heat is mean molecular kinetic energy"). Now the kind terms function as names for kinds, and "Water is $\mathrm{H}_{2} \mathrm{O}$ " expresses something like the following:

$$
\text { Waterkind }=\mathrm{H}_{2} \mathrm{O} \text {-kind }
$$

We saw earlier that, to deal with the trivialization objection, SP needs to commit itself to the view that kind terms designate, at worlds, functions from worlds to objects. So, on SP, kinds presumably should be identified with such functions.

On this reading of theoretical identities, then, the empirical discovery in (NAP1) would be an identity between kinds (construed as functions from worlds to extensions). From this, rigidity (in the sense of SP) would indeed deliver the necessity of the identity: if we can find out empirically that the kinds designated by A and B are identical, and we know that A and B are rigid (in SP's sense), we can conclude that the identity statement $\boldsymbol{A}=\boldsymbol{B}$ is necessary a posteriori. On the surface, then, SP seems to do the work required. But when we look at the case more closely, two problems arise.

Firstly, we should note that the kind terms are now really functioning as names for abstract objects. In other words, SP is succeeding in delivering the necessity of a posteriori identifications involving kind terms only by giving them a reading on which they are, in fact, singular terms. This is not what we were originally looking for: what we wanted is a notion of rigidity for general terms.

A second problem concerns the putative status of the identity as an empirically discovered fact. The identity mentioned in (NAP1) is now interpreted as holding between abstract objects, such as functions from worlds to objects. But how do we empirically observe such an identity? We cannot observe functions-they are causally inert. In the case of an identity statement between singular terms, things are fairly straightforward. We observe spatiotemporally located objects and find reason to suppose that the object with which we have been in causal contact on one occasion is in fact the same object with which we have had dealings at another.

\footnotetext{
${ }^{8}$ It has been brought to my attention that, contrary to what philosophers of language have for decades been taking for granted, "renate" and "cordate" do not mean "creature with a kidney" and "creature with a heart", but rather "reborn" and "heart-shaped". With due apologies to Eyja M. Brynjarsdóttir, I am nonetheless using the example because of its familiarity. And indeed, on SP, "renate" and "cordate" would be rigid on their OED-sanctioned meanings as well (though the putative co-extension is, admittedly, more far-fetched on this reading).
} 
But since kinds are, on SP, assumed to be abstract objects such as functions, we cannot have been in causal contact with them. The things we observe are members of kinds, not the kinds themselves.

Hence, the sense in which kind identities such as "Brontosaurus = Apatosaurus" are empirically discovered must be more indirect. We somehow reason to the identity, based on our empirical observations about members of the kinds in question. However, it is not immediately obvious how we do this. In the next section, I will take a closer look at this issue and claim that SP's notion of rigidity in fact plays no role at all in explaining the necessity of kind identities; the work expected of rigidity is really done by a notion I will introduce and label actualitydependence.

\section{Actuality-dependence}

So, what do we really discover when we discover that "Brontosaurus = Apatosaurus"? A full answer to this question would require settling the nature of biological species, and obviously I will not do that here. But some more general remarks will suffice to make my point. Whatever the precise nature of species, the discovery in question will have to be the discovery that the species-determining properties of the Brontosaurus and the Apatosaurus are one and the same. That is, we find that the feature-and presumably this will be something about a creature's genetics and/or lineage_-which makes something a brontosaur is the same as that which makes something an apatosaur.

This discovery presupposes that there are (or rather, have been) creatures which we have been calling "Brontosaurus", and ones we have been calling "Apatosaurus". Our discovery is not strictly speaking about kinds, but about features of the individuals that we have taken to fall under our kind terms. When we discover that we are dealing with just one species, not two, what we really discover is that the species-determining feature $\mathrm{F}_{\mathrm{B}}$, shared by (at least most of) the individuals we have taken to be brontosaurs is the same as the species-determining feature $\mathrm{F}_{\mathrm{A}}$, shared by (at least most of) the individuals we have taken to be apatosaurs.

This empirical discovery is not, by itself, the discovery of an identity between the kinds Brontosaurus and Apatosaurus. Hence, we cannot derive the necessary a posteriori truth of "Brontosaurus = Apatosaurus" from our empirical discovery and the observation that "Brontosaurus" and "Apatosaurus" are rigid in the sense of SP. But of course our discovery does, together with some additional premise $(s)$, entail that "Brontosaurus = Apatosaurus". But what additional premise(s) do we need? In particular, is there a (meta)semantic feature of the terms "Brontosaurus" and "Apatosaurus" that plays a central role in allowing us to reason from the empirical discovery to the identity?

I believe there is. The feature of "Brontosaurus" and "Apatosaurus" we need is that they are both actuality-dependent. ${ }^{9}$ As an informal characterisation, we can say

\footnotetext{
9 In an earlier paper (Haukioja 2006), I developed the notion of proto-rigidity, and claimed that it does much of the theoretical work usually expected of rigidity. The notion of actuality-dependence is naturally
} 
that for an actuality-dependent expression, the nature of the object or objects in its actual extension enters into determining its extension in non-actual contexts of interpretation. We let some feature of the actual extension of an actuality-dependent term fix its extension in non-actual worlds.

To get a more precise characterisation of actuality-dependence, and one which will help in making explicit the role it plays in explaining the necessity of a posteriori identifications, we need to introduce some new terminology. For any term, singular or general, something has to make it the case the term correctly applies, in the actual world, to the thing or things to which it in fact does apply. For different kinds of terms, the correct applicability may be determined in very different ways, and for many kinds of terms we do not have a widely agreed theory of how, exactly, this happens. Nonetheless, for any term T, something about us or our usage of $\mathrm{T}$ will determine a criterion that an object in the actual world has to satisfy in order to belong to T's extension. Such a criterion can be formulated as the specification of a role, which can be realized by different individuals or sets of individuals in different possible worlds. As putative examples of such roles, we might mention being an unmarried adult male, or being the individual at the origin of the causal chain of the appropriate type leading up to my use of the term "Arnold Schwarzenegger". For any term which in fact does possess conditions of correct application, such a role will have to exist, whether or not we can spell these roles out in detail.

Let us call such roles applicability roles: for any term $\mathrm{T}$, there is then an associated ${ }^{10}$ applicability role $\mathrm{R}_{\mathrm{T}}$, such that in the actual world, $\mathrm{T}$ applies to the thing(s) that play role $\mathrm{R}_{\mathrm{T}}$. Now we can define actuality-dependence:

(AD) $\mathrm{A}$ term $\mathrm{T}$ is actuality-dependent if and only if it applies, in non-actual worlds, to the thing(s) which possess the properties which realize ${ }^{11} \mathrm{R}_{\mathrm{T}}$ in the actual world. Otherwise, $\mathrm{T}$ is actuality-independent.

Typically, an actuality-independent term $\mathrm{T}$ will apply in non-actual worlds to the thing(s) which play the role $\mathrm{R}_{\mathrm{T}}$ in that world. For instance, the applicability role associated with "grandmother" will be (at least something very much like) being the mother of a parent. The extension of "grandmother" in non-actual worlds is not determined by another property that actual grandmothers happen to have: rather, it is determined by which individuals are mothers of parents in that world. Hence, "grandmother" is actuality-independent. The key idea here is that for any actualitydependent term T, the mechanisms for determining T's actual and counterfactual extensions will be different, and counterfactual extensions will depend on the nature of the things in T's actual extension. The extensions of actuality-independent terms are, by contrast, determined in a uniform way across possible worlds.

Footnote 9 continued

seen as a descendant of proto-rigidity: I am approaching things here from a slightly different angle, hoping to correct some of the shortcomings of the earlier paper.

${ }^{10}$ I am not claiming, or presupposing, that the role is semantically associated with the term, although with some actuality-dependent expressions it certainly is (for instance, rigidified descriptions).

${ }^{11} \mathrm{I}$ am here assuming that roles and realizers have to be distinct, in the sense that the property of playing role $R$ cannot be a realizer for $\mathrm{R}$. 
Applicability roles associated with natural kind terms will sound familiar to anyone who has thought about theories of reference for such terms. For example, the role $\mathrm{R}_{\mathrm{B}}$ for "Brontosaurus" might be being the same species as (most of) our Brontosaurus-samples, or maybe, being the same species as the creature(s) at the beginning of the causal chain leading up to my use of "Brontosaurus". The precise nature of this role will not matter for my purposes here. ${ }^{12}$ What does matter is that, whatever $\mathrm{R}_{\mathrm{B}}$ precisely turns out to be, in counterfactual circumstances the extension of "Brontosaurus" is not determined by what (if anything) plays role $\mathrm{R}_{\mathrm{B}}$ in that world. Rather, the feature $\mathrm{F}$ that actually makes something play role $\mathrm{R}_{\mathrm{B}}$ determines the correct application of "Brontosaurus" in non-actual worlds. Similar remarks hold for "Apatosaurus": its applicability in non-actual worlds is determined by the actual realizers of a similar but distinct role, $\mathrm{R}_{\mathrm{A}}$.

Now we are in a position to see how actuality-dependence is involved in establishing the necessity of identity statements. The empirical discovery discussed earlier was the discovery that the distinct roles, $\mathrm{R}_{\mathrm{A}}$ and $\mathrm{R}_{\mathrm{B}}$, are actually realized by just one property, F. This discovery, together with the actuality-dependence of the two species terms, entails the necessity of "Brontosaurus = Apatosaurus", read as a quantified biconditional: at any possible world, the feature $F$ which makes something belong in the extension of "Brontosaurus" also makes it belong in the extension of "Apatosaurus", and vice versa. Hence, the quantified biconditional "All and only brontosaurs are apatosaurs" is necessary, although it is a posteriori.

The explanation generalizes: we can reformulate the schema for deriving the necessity of identification statements as (NAP*):

(NAP1*) Discover empirically that, in the actual world, the applicability roles associated with terms A and B are realized by the same property.

(NAP2*) Note that A and B are actuality-dependent.

(NAP3*) Reason from (NAP1*) and (NAP2*) that $\boldsymbol{A}=\boldsymbol{B}$ is necessary.

The identity in (NAP3*) is to be read as a quantified biconditional, $\forall x(x$ is $\mathrm{A} \leftrightarrow x$ is $\mathrm{B})$.

When $\mathrm{A}$ and $\mathrm{B}$ are general terms, we could-at least with a suitable understanding of the metaphysics of kinds-go on to derive the identity between kinds (as abstracta) from (NAP3*), and then use the SP account of rigidity to show its necessity. However, all the interesting theoretical work has already been done by actuality-dependence, so the result is of minor importance. The fundamental (meta)semantic feature responsible for the necessity of a posteriori identity statements is actuality-dependence, not the account of rigidity put forward by SP. ${ }^{13}$

The necessity of identity statements involving singular terms can also be explained by (NAP*). Let us take a look at "Hesperus is Phosphorus": in (NAP1*)

\footnotetext{
12 "Being the same species as" is of course similarly in need of further elaboration-but these details will also not be relevant for the points I am making in this paper.

13 For quantified conditionals such as "Tigers are animals", the schema works in a slightly different fashion: the empirical discovery is the finding that anything possessing the realizer of the applicability role for "tiger" also possesses a realizer of the applicability role for "animal" (but not vice versa). Or, to put it more informally, whatever it is that makes something a tiger also makes it an animal (but not the other way around).
} 
we discover that the applicability roles associated with the two names are realized by the same property. It is not obvious what these roles are, precisely: among the candidates we will be likely to find roles such as being the brightest star in the evening/morning sky, or being the object at the beginning of the causal chain of the appropriate type leading up to my use of "Hesperus"/.Phosphorus". These details are not important here: whatever the applicability roles are, in (NAP1*) we discover that these roles are actually realized by one property, that of being Venus.

In (NAP2*) we note that "Hesperus" and "Phosphorus" are actuality-dependent. In fact, all proper names are actuality-dependent: the identity of a name's actual referent determines its referent in non-actual worlds. Or, to put it in terms of applicability roles and their realizers, assuming roughly a Kripkean theory of reference: the individual which actually plays the role of being at the beginning of a causal chain (of the appropriate type) leading up to my use of a name is the name's referent in all worlds (in which it exists). In the case of singular terms, actualitydependence and de iure rigidity coincide. ${ }^{14}$

\section{Is rigidity actuality-dependence?}

The idea that a feature like actuality-dependence is somehow relevant to the questions surrounding rigidity and the necessary a posteriori is hardly surprising. Indeed, Putnam's claim that natural kind terms "have an unnoticed indexical component" (Putnam 1975, p. 234) can already be seen as hinting in this direction, ${ }^{15}$ and many authors have pointed out the importance of local samples in the determination of reference for natural kind terms. However, I am not aware of any earlier work where this feature of natural kind terms is directly made use of in explaining the necessity of a posteriori identifications. If I am right, and actualitydependence really can do this theoretical work (that is, do the work we usually expect of rigidity), it is natural to wonder whether we should conclude that rigidity is actuality-dependence-that is, that actuality-dependence should be seen as an elucidation of our notion of rigidity, a rival to the essentialist view and SP.

\footnotetext{
${ }^{14}$ The explanation of a posteriori necessity by relying on applicability roles and their realizers is in some ways similar to the one given by Glüer and Pagin in recent papers (Glüer and Pagin 2006, forthcoming), where they claim that proper names and natural kind terms have two separate intensions, one latching on to manifest properties, the other to underlying properties. As much as I admire their technical framework of relational modality, I believe my account is superior in one respect, in that it is more widely applicable. On my account, all the explanation is done on the level of metasemantics rather than semantics: I am not making any presuppositions about what the semantic values of names or kind terms are. All that matters is that the reference of a name or the extension of a natural kind term is determined in one way for the actual world and in another for non-actual worlds. This assumption could, at least in principle, be accommodated in a variety of different semantic frameworks. This is a clear advantage: one can believe that there are necessary a posteriori identity statements whether one is a Millian, a two-factor theorist, or even an actualised-description theorist. What matters is the dual nature of reference-determination, not the issue of what semantic values names and natural kind terms are assigned.

15 Putnam goes on to characterize his view as follows: "Water at another time or in another place or even in another world has to bear the relation same $_{\mathrm{L}}$ to our 'water' in order to be water" (ibid., emphasis in the original) — this is very naturally read as pointing to something like actuality-dependence.
} 
There are enough connections between actuality-dependence and our usual expectations regarding rigidity to make such an identification seem quite attractive, at least initially. Actuality-dependence does not suffer from trivialization or overgeneralization problems: as we have seen above, natural kind terms are actuality-dependent, while most other general terms (such as "bachelor" or "grandmother") are not. Moreover, terms such as "red" and "hot", though not natural kind terms, are arguably actuality-dependent. ${ }^{16}$

However, there is also a major problem for seeing actuality-dependence as an elucidation of the notion of rigidity: terms such as " $\mathrm{H}_{2} \mathrm{O}$ " are actuality-independent. In explaining the necessary a posteriori status "Water is $\mathrm{H}_{2} \mathrm{O}$ ", we only need to rely on the actuality-dependence of "water". The applicability of "water" is determined, in non-actual worlds, by the actual realizer of the applicability role of water, and if chemistry is to be trusted, " $\mathrm{H}_{2} \mathrm{O}$ " is a description of this realizer. The applicability of " $\mathrm{H}_{2} \mathrm{O}$ " in non-actual worlds is determined by the applicability role of " $\mathrm{H}_{2} \mathrm{O}$ " (roughly, that is, by being a compound molecules of which consist of two hydrogen atoms and one oxygen atom), and not by any actual realizer of this role. So " $\mathrm{H}_{2} \mathrm{O}$ " is actuality-independent; yet it is usually assumed to be rigid. ${ }^{17}$

What should we conclude, then? I can see three ways to go from here. First, we could let SP have the label "rigidity" - after all, given their assumption about what general terms designate, SP follows directly from Kripke's definition of rigid designation. However, on this view we must keep in mind that it is actualitydependence, not rigidity as conceived of by SP, that is doing the work in explaining the necessity of true identity statements. Second, we could equate rigidity with actuality-dependence. This option would require us to bite the bullet on " $\mathrm{H}_{2} \mathrm{O}$ " and related expressions, but at the same time it would hold on to the link between rigidity and the necessary a posteriori. Finally, we could simply conclude that neither SP nor actuality-dependence fulfils our criteria for a satisfactory notion of rigidity, and keep looking for a better one. I am not very optimistic about the last option; the choice between the first two looks to me like a fairly uninteresting terminological question. But whichever of them we choose to take, the notion of actuality-dependence will play a central theoretical role. ${ }^{18}$

\footnotetext{
${ }^{16}$ In fact, discussions about whether colour terms are rigid really seem to be about whether they are actuality-dependent-that is, whether their extensions in non-actual worlds are determined by the properties which in the actual world make objects look red to normal observers in normal conditions (or something very similar to this).

17 Another-in my view less crucial-disanalogy between actuality-dependence and our usual expectations of rigidity concerns numerals and other designators for abstracta. Numerals are quite often given as examples of rigid expressions, but it is obvious that they are not actuality-dependent.

18 An interesting question, which I will not even try to answer here in detail, is why we have actualitydependent terms in the first place. Why do some terms have this dual kind of extension-determination? Some hints at a possible explanation can be found in the literature on a related phenomenon in psychology. Psychological essentialism is an empirically well-established hypothesis according to which humans have, concerning some categories, an implicit commitment to the category having an underlying, non-manifest essence (for an overview, see for example Gelman 2004). It appears that our actualitydependent terms express concepts for which psychological essentialism holds. An attractive explanation for psychological essentialism, in turn, relies on evolutionary considerations: essentialist concepts would presumably have conferred an evolutionary advantage on their possessors, based on their stronger inductive potential. The use of actuality-dependent terms may, then, have also enabled us to better
} 
Acknowledgments This paper grew out of material presented at the INPC conference in Moscow, Idaho (2008), at the XXII World Congress of Philosophy in Seoul (2008), at the ECAP conference in Krakow (2008), at the Pacific APA meeting in Vancouver (2009), as well as seminars and colloquia at the University of Turku, SUNY Buffalo and University of Minnesota. I am grateful to the audiences at all these events; I wish to especially thank Eyja M. Brynjarsdóttir, Sam Cowling, Michael Devitt, Michael O'Rourke, and Benjamin Schnieder for detailed comments on earlier written versions. The final version of the paper also benefited substantially from excellent comments and suggestions made by an anonymous referee for this journal. This work was financially supported by the Academy of Finland (grant 207129).

Open Access This article is distributed under the terms of the Creative Commons Attribution Noncommercial License which permits any noncommercial use, distribution, and reproduction in any medium, provided the original author(s) and source are credited.

\section{References}

Cook, M. (1980). If 'cat' is a rigid designator, what does it designate? Philosophical Studies, 37, 61-64. Devitt, M. (2005). Rigid application. Philosophical Studies, 125, 139-165.

Gelman, S. A. (2004). Psychological essentialism in children. Trends in Cognitive Science, 8, 404-409. Glüer, K., \& Pagin, P. (2006). Proper names and relational modality. Linguistics \& Philosophy, 29, 507-535.

Glüer, K. \& Pagin, P. (forthcoming). General terms and relational modality. Nôus.

Gómez-Torrente, M. (2006). Rigidity and essentiality. Mind, 115, 227-260.

Haukioja, J. (2006). Proto-rigidity. Synthese, 150, 155-169.

Kripke, S. (1980). Naming and necessity. Cambridge: Harvard University Press.

LaPorte, J. (2000). Rigidity and kind. Philosophical Studies, 97, 293-316.

LaPorte, J. (2004). Natural kinds and conceptual change. Cambridge: Cambridge University Press.

LaPorte, J. (2006). Rigid designators for properties. Philosophical Studies, 130, 321-336.

Linsky, B. (2006). General terms as rigid designators. Philosophical Studies, 128, 655-667.

López de Sa, D. (2007). Rigidity, general terms, and trivialization. Proceedings of the Aristotelian Society, 56, 117-123.

López de Sa, D. (2008). The over-generalization problem: predicates rigidly signifying the 'unnatural'. Synthese, 163, 263-272.

Martí, G. (2004). Rigidity and general terms. Proceedings of the Aristotelian Society, 104, 129-146.

Martí, G., \& Martínez, P. (2007). General terms and non-trivial rigid designation. In C. Martínez (Ed.), Current topics in logic and analytic philosophy (pp. 103-116). Santiago de Compostela: Universidad de Santiago de Compostela.

Putnam, H. (1975). The meaning of 'meaning'. In Philosophical papers (vol. 2, pp. 215-271). Cambridge: Cambridge University Press.

Salmon, N. (1982). Reference and essence. Oxford: Blackwell.

Salmon, N. (2005). Are general terms rigid? Linguistics \& Philosophy, 28, 117-134.

Schwartz, S. (2002). Kinds, general terms, and rigidity: A reply to LaPorte. Philosophical Studies, 109, $265-277$.

Soames, S. (2002). Beyond rigidity. The unfinished semantic agenda of naming and necessity. Oxford: Oxford University Press.

Footnote 18 continued

express successful inductive statements, by allowing us to focus not on manifest properties, but on the underlying properties which are causally more relevant. 